\title{
Fault Diagnosis of an Air-Conditioning System Using LS-SVM
}

\author{
Mahendra Kumar ${ }^{1}$ and I.N. $\operatorname{Kar}^{2}$ \\ ${ }^{1}$ Working as Deputy Chief Electrical Engineer in Northern Railway, Delhi-110001 \\ ${ }^{2}$ Department of Electrical Engineering, Indian Institute of Technology, Delhi-110016 \\ mahendray71@gmail.com, ink@ee.iitd.ac.in.
}

\begin{abstract}
This paper describes fault diagnosis of an air-conditioning system for improving reliability and guaranteeing the thermal comfort and energy saving. To achieve this goal, we proposed a technique which is model based fault diagnosis technique. Here, a lumped parameter model of an air-conditioning system is considered and then characteristics of twelve faults are investigated in an airconditioning system provided in passenger coach of an Indian Railway. Based on the variations of the system states under normal and faulty conditions of different degrees, the faults can be detected efficiently by using residual analysis method. The residual code is obtained through simple threshold testing of residuals, which are the output of a general scheme of residual generators. The pattern of residual is classified by using multi-layer LS-SVM classification. The diagnosis results show that LS-SVM classifier is effective with very high accuracy.
\end{abstract}

Keywords: LS-SVM, Residuals generator, Air-Conditioning System, FDD.

\section{Introduction}

The reliability of an air-conditioning (AC) system has a prime importance as failure of AC system can lead to occupant's discomfort, higher health and safety risks. The faulty and non optimal operation of an AC system wastes an estimated $15 \%$ to $30 \%$ of energy used in commercial building. Hence, fault detection and diagnosis (FDD) methods for an air-conditioning system is an important area. The main purpose of FDD is, to detect, locate and if possible, predict the presence of the defects causing faulty operation. A fault is detected when the observed behaviour of a system differs from the expected behaviour by some threshold. The expected behaviour of the system is expressed in a model whether physical, statistical or fuzzy. In this paper we have used model based on physical laws. The fault diagnosis has been done by residual analysis. A residual is often a time varying signal that is used as a fault detector. Normally, the residual is designed to be zero (or small in a realistic case where process is subjected to noise and the model is uncertain) in fault free case and deviate significantly from zero when fault occurs. So fault is identified by comparing residual with threshold value. The sequence of residual is identified using LS-SVM. The least square support vector machine (LS-SVM) is used for classification of different residuals pattern. In this paper twelve faults have been considered for diagnosis and 
eight residuals have generated for fault classification. Fault diagnosis of an air conditioning system has been the subject of many studies during the past decades. Generally, the fault detection and diagnosis (FDD) methods can be divided into three types [7]: the feature-based method, the model-based method, and a combination of both. Although these methods have been applied in a number of industrial processes with good performance, their application in the HVAC system is still at the research stage in laboratories [6]. The main contributions of this paper are as follows:

1. A model based fault diagnosis schemes have been developed by using residuals for diagnosing major air-conditioning faults.

2. The fault diagnosis is done by residuals pattern classification using LSSVM as classifier.

3. The residuals of the fault are generated by using an air-conditioning system provided in passenger coach of an Indian Railway and simulation of the proposed fault diagnosis scheme has been done.

\section{Design of Model Based Fault Diagnosis Scheme}

The first requirement of model based fault diagnosis scheme is model of the plant which represents expected behaviour of the system. In this paper we have considered lumped parameter model of an air conditioning system. In model based fault diagnosis scheme the output and control input of the plant and the output and control input of fault free model is compared and residuals are generated. A pattern of residuals is generated for a fault and in this paper we have proposed fault diagnosis scheme using LS-SVM as shown in Fig.1.

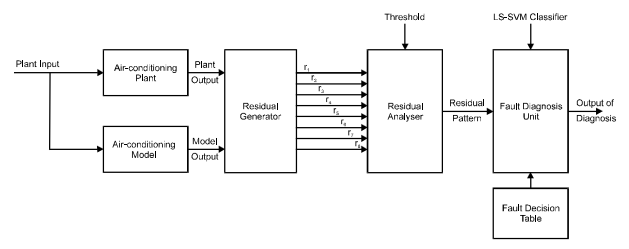

Fig. 1. The Block diagram of model based fault diagnosis scheme

The performance of the proposed fault diagnosis scheme is investigated on a lumped parameter model [1] in which complete dynamical model of an airconditioning system has been considered. This dynamic model has been developed based on principles of mass and energy conservation. The dynamic mathematical model can be represented in state space form.

$$
\begin{gathered}
\dot{x}=g(x, u, d) \\
y=h(x, u)
\end{gathered}
$$

where $x=\left[\begin{array}{llllllllllll}x_{1} & x_{2} & x_{3} & x_{4} & x_{5} & x_{6} & x_{7} & x_{8} & x_{9} & x_{10} & x_{11} & x_{12}\end{array}\right]^{\prime}$ are the states. Control input vector is given by $\mathrm{u}$, disturbance vector is $\mathrm{d}$ and output vector is $\mathrm{y}$ $u=\left[\begin{array}{llll}u_{1} & u_{2} & u_{3} & u_{4}\end{array}\right]^{\prime} \quad d=\left[\begin{array}{llllll}d_{1} & d_{2} & d_{3} & d_{4} & d_{5}\end{array}\right]^{\prime} \quad y=\left[\begin{array}{llll}y_{1} & y_{2} & y_{3} & y_{4}\end{array}\right]^{\prime}$ 
The details of the model is presented in [1]. The general scheme of residual generator is given below:

Consider a state-space realization of the continuous-time plant model given by

$$
\begin{aligned}
\dot{x}(t)=A x(t)+B u(t)+E d(t) \\
y(t)=C x(t)+D u(t)+F d(t)
\end{aligned}
$$

Where $d(t)$ is the fault vector to be detected. In general, $d(t)$ consists of faults which may occur in the actuators, the plant dynamics (components), or the sensors. The initial state $x(0)$ is assumed to be zero. Taking the s-transform of Eq.(3)-(4) gives

$$
y(s)=G_{u}(s) u(s)+G_{d}(s) d(s)
$$

where $G_{u}(s)=C(s I-A)^{-1} B+D$ and $G_{d}(s)=C(s I-A)^{-1} E+F$

A residual generator can be expressed in a general form as

$$
r(s)=F(s) u(s)+H(s) y(s)
$$

with the properties that for all $u(s)$,

i) $r_{i}(s)=0$ if $d_{i}(s)=0$ and

ii) $r_{i}(s) \neq 0$ if $d_{i}(s) \neq 0, i=1,2, \ldots \ldots \ldots . ., q$

where $F(s)$ and $H(s)$ are stable and proper transfer matrices that are realizable in a real system. When no failure occurs, all the residuals are equal or close to zero. In presence of $i$-th failure signal, residual signal will become distinguishably nonzero.

\section{Fault Detection Based on Residual Analysis}

\subsection{Residual Analysis}

Residual analysis is an easy way to study deviation of actual value from normal value. The residual can be computed by taking the difference between state variable under normal and faulty condition. Figs.2-3 show the residuals for various faults. In the above simulation the threshold are set at $\pm 2{ }^{\circ} \mathrm{C}, \pm 0.5$ bar, \pm 0.5 bar, $-1.25^{\circ} \mathrm{C},-5 \%$ for evaporator wall temperature, evaporator pressure, condenser pressure, coach air temperature and relative humidity respectively. If the residual between the system output and actual output exceed the threshold, a fault alarmed.

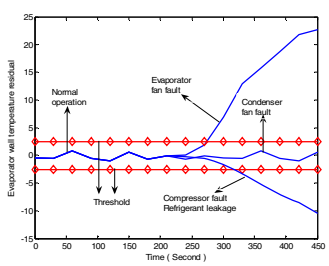

Fig. 2. Evaporator wall temperature residual

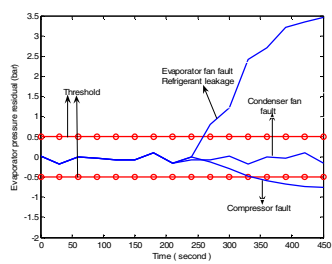

Fig. 3. Evaporator pressure residual 


\subsection{Fault Decision Table}

In this section we will discuss different residual for different faults. The residual patterns for different faults are shown in Table 1. These residuals have been generated by using an air-conditioning unit provided in Indian Railway passenger coach faults.

$r_{1}=$ Evaporator wall temperature, $r_{2}=$ Thermal space temperature, $r_{3}=$ Absolute humidity of thermal space, $r_{4}=$ Evaporator Pressure, $r_{5}=$ Condenser pressure, $r_{6}=$ Condenser wall temperature, $r_{7}=$ Speed of compressor, $r_{8}=$ Air flow rate.

Fault 0: No fault, Fault 1: Evaporator fan speed less/ Air filters are choked wall temperature sensor defective. Fault 2: Condenser fan speed less. Fault 3: Compressor defective. Fault 4: Refrigerant leakage. Fault 5: Evaporator wall temperature sensor defective. Fault 6: Thermal space temperature sensor defective. Fault 7: Relative humidity sensor defective. Fault 8: Evaporator pressure sensor defective. Fault 9: Condenser pressure sensor defective. Fault 10: Condenser temperature sensor defective. Fault 11: Compressor speed sensor defective. Fault 12: Air flow rate sensor defective.

Table 1. Fault symptoms

\begin{tabular}{|c|c|c|c|c|c|c|c|c|}
\hline \multirow{2}{*}{$\begin{array}{l}\text { Fault } \\
\text { modes }\end{array}$} & \multicolumn{8}{|c|}{ Residuals } \\
\hline & $r_{1}$ & $r_{2}$ & $r_{3}$ & $r_{4}$ & $r_{5}$ & $r_{6}$ & $r_{7}$ & $r_{8}$ \\
\hline 0 & $\rightarrow$ & $\rightarrow$ & $\rightarrow$ & $\rightarrow$ & $\rightarrow$ & $\rightarrow$ & $\rightarrow$ & $\rightarrow$ \\
\hline 1 & $\downarrow$ & $\uparrow$ & $\uparrow$ & $\downarrow$ & $\downarrow$ & $\rightarrow$ & $\rightarrow$ & $\downarrow$ \\
\hline 2 & $\rightarrow$ & $\uparrow$ & $\uparrow$ & $\rightarrow$ & $\uparrow$ & $\uparrow$ & $\rightarrow$ & $\rightarrow$ \\
\hline 3 & $\uparrow$ & $\uparrow$ & $\uparrow$ & $\uparrow$ & $\downarrow$ & $\downarrow$ & $\downarrow$ & $\rightarrow$ \\
\hline 4 & $\uparrow$ & $\rightarrow$ & $\rightarrow$ & $\uparrow$ & $\uparrow$ & $\uparrow$ & $\rightarrow$ & $\rightarrow$ \\
\hline 5 & $\uparrow \downarrow$ & $\rightarrow$ & $\rightarrow$ & $\rightarrow$ & $\rightarrow$ & $\rightarrow$ & $\rightarrow$ & $\rightarrow$ \\
\hline 6 & $\rightarrow$ & $\uparrow \downarrow$ & $\rightarrow$ & $\rightarrow$ & $\rightarrow$ & $\rightarrow$ & $\rightarrow$ & $\rightarrow$ \\
\hline 7 & $\rightarrow$ & $\rightarrow$ & $\uparrow \downarrow$ & $\rightarrow$ & $\rightarrow$ & $\rightarrow$ & $\rightarrow$ & $\rightarrow$ \\
\hline 8 & $\rightarrow$ & $\rightarrow$ & $\rightarrow$ & $\uparrow \downarrow$ & $\rightarrow$ & $\rightarrow$ & $\rightarrow$ & $\rightarrow$ \\
\hline 9 & $\rightarrow$ & $\rightarrow$ & $\rightarrow$ & $\rightarrow$ & $\uparrow \downarrow$ & $\rightarrow$ & $\rightarrow$ & $\rightarrow$ \\
\hline 10 & $\rightarrow$ & $\rightarrow$ & $\rightarrow$ & $\rightarrow$ & $\rightarrow$ & $\uparrow \downarrow$ & $\rightarrow$ & $\rightarrow$ \\
\hline 11 & $\rightarrow$ & $\rightarrow$ & $\rightarrow$ & $\rightarrow$ & $\rightarrow$ & $\rightarrow$ & $\uparrow \downarrow$ & $\rightarrow$ \\
\hline 12 & $\rightarrow$ & $\rightarrow$ & $\rightarrow$ & $\rightarrow$ & $\rightarrow$ & $\rightarrow$ & $\rightarrow$ & $\uparrow \downarrow$ \\
\hline
\end{tabular}

\section{Fault Diagnosis Using LS-SVM Classifier}

The patterns of residuals given in Table 1 can be classified by using some classifier. In this paper a LSSVM classifier is designed to diagnose air-conditioning faults. In case of LS-SVM, original Vapnik's SVM classifier formulation has been modified as follows:

$$
\min _{w, b, e} \mathfrak{I}(w, e)=\frac{1}{2} w^{T} w+\gamma \frac{1}{2} \sum_{k=1}^{N} e_{k}^{2}
$$

subject to the equality constraints

$$
y_{k}\left[w^{T} \varphi\left(x_{k}\right)+b\right]=1-e_{k}, \quad \mathrm{k}=1, \ldots ., \mathrm{N} .
$$


The important differences with standard SVMs are the equality constraints and the sum squared error term. Then the Lagrangian of the system can be constructed like:

$$
\ell(w, b, e ; \alpha)=\mathfrak{I}(w, e)-\sum_{k=1}^{N} \alpha_{k}\left\{y_{k}\left[w^{T} \varphi\left(x_{k}\right)+b\right]-1+e_{k}\right\}
$$

where $\alpha_{k}$ are the Lagrange multipliers. The condition of optimality can be given by

$$
\begin{aligned}
& {\left[\begin{array}{cc}
0 & Y^{T} \\
Y & \vartheta+\gamma^{-1} I
\end{array}\right]\left[\begin{array}{l}
b \\
\alpha
\end{array}\right]=\left[\begin{array}{l}
0 \\
I
\end{array}\right] } \\
& Z=\left[\varphi\left(x_{1}\right)^{T} y_{1}, \ldots \ldots ., \varphi\left(x_{N}\right)^{T} y_{N}\right] \\
& Y= {\left[y_{1}, \ldots \ldots \ldots y_{N}\right], I=[1, \ldots \ldots .1] } \\
& \alpha=\left[\alpha_{1}, \ldots \ldots . \alpha_{N}\right] \text { and } \vartheta=\mathrm{ZZ}^{\mathrm{T}} \\
& \text { or, } \quad \vartheta_{k l}=y_{k} y_{l} \varphi\left(x_{k}\right)^{T} \varphi\left(x_{l}\right)=y_{k} y_{l} K\left(x_{k}, x_{l}\right)
\end{aligned}
$$

$K(.,$.$) is the kernel function. LS-SVM classifier is constructed as follows:$

$$
y(x)=\operatorname{sign}\left[\sum_{k=1}^{N} \alpha_{k} y_{k} K\left(x, x_{k}\right)+b\right] .
$$

\section{Results and Discussions}

In this paper, in order to achieve better on-line FDD performance, a heuristic algorithm using LSSVM is used to classify 12 different faults. Since the LS-SVM classifier presented in Fig. 4 can only be used to deal with three class case, a multilayer LS-SVM frame work has to be designed for the FDD problem with various

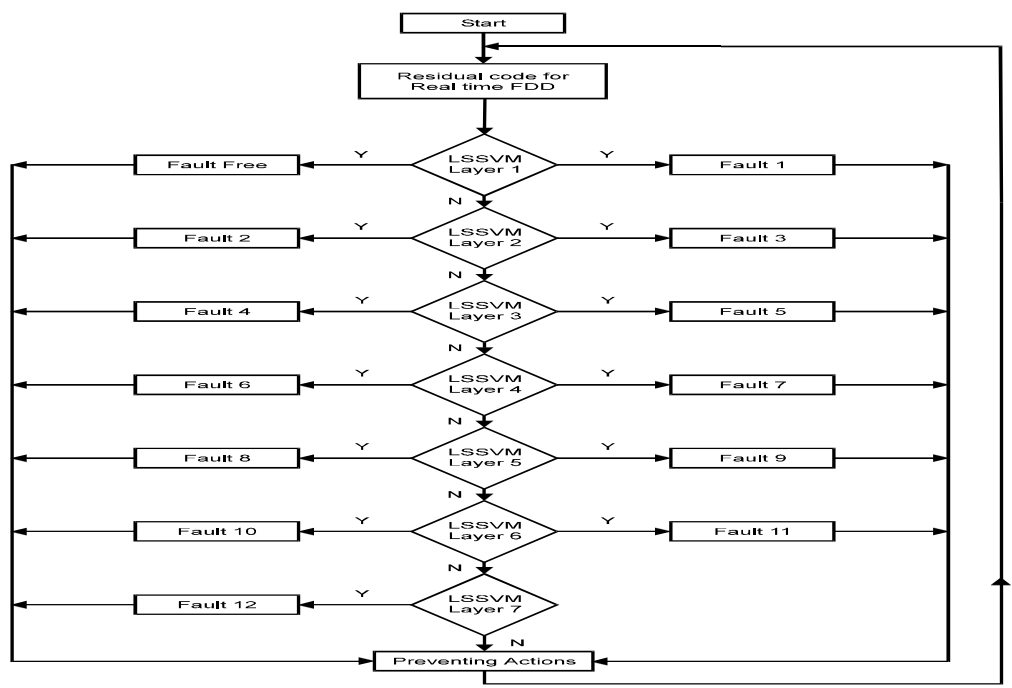

Fig. 4. Flow chart of the seven -layer LS-SVM classifier 
faulty positions. It is interesting to know that for conventional neural network classifiers, the architecture and neuron number must be properly designed to achieve high classification accuracy. But for the SVM classifier, the key is to choose a proper kernel function. In general, linear function, polynomial function, radial basis function (RBF), sigmoid function etc. can be adopted as the kernel function. In this paper, polynomial function is used in the designed LSSVM classifier. as it has excellent performance in many applications.

The performance of this FDD method is measured in terms of diagnosis accuracy. The steady state variable data between 4 and $10 \mathrm{~h}$ are used to build the seven layer SVM classifier, the data with in the threshold under the normal condition indicate fault free, and the data beyond the threshold indicate faults 1-12. For each normal/ faulty condition, $256 \times 12$ groups of sample data are used, in which 256 groups are used for each fault degree. Therefore a total of 8 (condition) $\times 256 \times 12$ (samples) are collected. The LSSVM classifier has been tested by using data other than training data, in absence as well as presence of noise. It has been found that LSSVM classifier is able to diagnose all twelve faults.

\section{Conclusions}

This paper proposes a fault diagnosis schemes having model based approach for diagnosing major faults in an air-conditioning system. In model based fault diagnosis using LS-SVM classifier very good diagnosis accuracy has been achieved.

(i) The most essential condition for achieving good accuracy is that model should be accurate. The inaccuracy in model will directly reduce fault diagnosis accuracy.

(ii) Fault diagnosis accuracy also depends upon threshold selection.

(iii)The practical systems have always noises and robustness of these fault diagnosis schemes against sensor noises needs to be examined.

\section{References}

[1] Kumar, M., Kar, I.N., Ray, A.: State Space based Modelling and Performance Evaluation of air-conditioning system. International Journal of HVAC \& R Research 14(5) (September 2008)

[2] Liang, J., Du, R.: Model-based fault detection and diagnosis of HVAC systems using Support Vector Machine method. International Journal of refrigeration 30, 1104-1114

[3] Pau-Lo-Hsu, Lin, K.-L., Shen, L.-C.: Diagnosis of Multiple Sensor and Actuator Failures in Automotive Engines. IEEE Transactions on Vehicular Technology 44(4) (November 1995)

[4] Stoecker, W.F., Jones, J.W.: Refrigeration \& Air conditioning. McGraw-Hill Book Company, New York (1982)

[5] The Math Works, Inc., MATLAB. The Math Works, Inc., Natick, Massachusetts (2007)

[6] Katipamula, S., Brambley, M.R.: Methods for fault detection, diagnostics, and prognostics for building systems-a review, part I. International Journal of HVAC\&R Research 11, 3 25 (2005)

[7] Du, R.: Monitoring and Diagnosis of Sheet Metal Stamping Processes. In: Gao, R., Wang, L.H. (eds.) Condition-based Monitoring and Control for Intelligent Manufacturing. Springer, New York (2005) 